\title{
Introduction : la dimension argumentative du discours - enjeux théoriques et pratiques
}

\section{Ruth Amossy}

\section{(2) OpenEdition \\ 1 Journals}

Édition électronique

URL : http://journals.openedition.org/aad/2560

DOI : $10.4000 /$ aad. 2560

ISSN : $1565-8961$

Éditeur

Université de Tel-Aviv

\section{Référence électronique}

Ruth Amossy, «Introduction : la dimension argumentative du discours - enjeux théoriques et pratiques », Argumentation et Analyse du Discours [En ligne], 20 | 2018, mis en ligne le 15 avril 2018, consulté le 23 septembre 2019. URL : http://journals.openedition.org/aad/2560 ; DOI : 10.4000/aad. 2560

\section{Ce document a été généré automatiquement le 23 septembre 2019}

\section{c) (i) (9)}

Argumentation \& analyse du discours est mis à disposition selon les termes de la licence Creative Commons Attribution - Pas d'Utilisation Commerciale - Pas de Modification 4.0 International. 


\title{
Introduction : la dimension argumentative du discours - enjeux théoriques et pratiques
}

\author{
Ruth Amossy
}

\section{L'argumentation entre conception restreinte et conception étendue}

1 Le présent dossier se propose de revenir sur la notion aussi influente que discutée de "dimension argumentative » mise en place dans la théorie de l'argumentation dans le discours (Amossy 2012 [2000], 2005), mais aussi développée dans d'autres travaux, en particulier par Rabatel $\left(2004,2014,2016^{1}\right)$ sous le nom d'« argumentation indirecte ». Il s'agit à la fois d'en tester la rentabilité, et de revenir sur les problèmes théoriques qu'elle a soulevés. Si elle s'est retrouvée au centre d'un débat, c'est en effet parce qu'elle met en jeu des questions fondamentales concernant d'une part la définition (et les frontières) de l'argumentation, et d'autre part les outils nécessaires à son analyse.

2 En effet, la dimension argumentative marque l'écart qui sépare une conception restreinte et une conception large ou étendue de l'argumentation (il va de soi que restreinte n'a ici aucun sens péjoratif). Chacune repose sur une vision différente de la pratique de l'argumentation, et de la discipline qui en traite. Toutes deux sont bien sûr légitimes et chacune d'elle comporte ses avantages et ses inconvénients ${ }^{2}$ - comme nous le verrons par la suite. La conception restreinte entend limiter l'argumentation au déploiement d'un discours qui use d'arguments pour prouver le bien-fondé d'une thèse ; elle l'étudie dans sa singularité en le différenciant de tout ce qui n'en relève pas de façon stricte. Dans ce sens, elle est donc exclusive. La conception étendue est inclusive : elle englobe l'argumentation comprise au sens strict et la place au cœur de ses préoccupations; mais elle la situe au centre d'un continuum qui comprend à l'une de ses extrémités la polémique comme confrontation violente de thèses antagonistes, 
et de l'autre une orientation des façons de penser et de voir, de questionner et de problématiser, qui ne s'effectue pas par la voie du raisonnement formel.

3 Avant d'engager plus avant la réflexion, précisons la nature de ces deux options. Les théories de l'argumentation restreinte se focalisent sur le logos, y voyant avant tout un partage de la raison effectué sur la base d'arguments logiquement valides menant de prémisses partagées à une position présentée comme vraie, ou tout au moins comme raisonnable. Même si elles sont très diverses, les théories de l'argumentation qui relèvent de cette approche mettent l'accent sur (1) le raisonnement (2) en situation de communication (3) concernant une question controversée (4) menant à une thèse (5) ayant recours à des procédures argumentatives formalisables. Sans doute peuvent-elles faire place au pathos - comme le montrent bien les travaux de Plantin (2011) ou de Micheli (2010] -, mais l'existence de l'argumentation dépend du logos qui en constitue l'ossature.

4 La notion de dimension argumentative a été au contraire avancée pour penser des formes d'argumentation alternatives qui dépassent les formes canoniques. En se penchant sur l'éloge funèbre (et donc l'épidictique), le témoignage, la description, les textes dits d'information, la conversation familière, la lettre, le récit littéraire, et bien d'autres, elle montre qu'ils argumentent à leur façon. Ils le font dans le sens où ils tentent de faire partager des opinions, des vues, des questionnements, à travers des procédures discursives qui ne sont pas des arguments en forme.

5 Sans doute n'est-il pas inutile de rappeler une fois de plus que la notion de dimension argumentative ou argumentation indirecte, et la conception étendue de l'argumentation dont elle participe, s'inspirent des travaux précurseurs de Grize qui dans le chapitre «L'omniprésence de l'argumentation » de Logique et langage (1990:40), écrivait: "Argumenter renvoie à justifier, expliquer, étayer [...] Mais il est aussi possible de concevoir l'argumentation d'un point de vue plus large et de l'entendre comme une démarche qui vise à intervenir sur l'opinion, l'attitude, voire le comportement de quelqu'un ». Ce que Plantin résume par: "Argumenter, c'est métaphoriquement "orienter" le regard» $(2016: 78)$.

6 On reprendra ici brièvement les divers aspects de cette conception étendue, les problèmes qui en découlent, et les arguments qui peuvent jouer en sa faveur.

\section{L'argumentation comme dimension constitutive du discours}

7 En un premier temps, et dans sa forme la plus extrême, la notion de dimension argumentative situe l'argumentativité au cœur même du discours. Elle est de ce point de vue le pendant discursif des conceptions issues de Ducrot (Anscombre \& Ducrot 1988), qui situent l'argumentation dans la langue. De ce fait disparaît la séparation traditionnelle entre une discipline qui traite du discours (l'analyse du discours ou AD) et une autre qui traite de l'argumentation (la théorie de l'argumentation) : il s'agit d'analyser dans leur matérialité discursive les modalités de l'argumentation directe aussi bien qu'indirecte. La première se fonde sur des schèmes argumentatifs qui nécessitent une mise en mots; la seconde s'élabore dans l'épaisseur du discours endehors de schèmes argumentatifs repérables (Amossy 2005, 2009). 
C'est dans cette perspective que l'édition de 2010 (et 2012) de L'argumentation dans le discours ajoute dans l'introduction un passage qui fait de l'argumentativité un trait constitutif du discours, à côté de l'énonciation et de la subjectivité (Benveniste 1966, 1974, Kerbrat-Orecchioni 1980), du dialogisme (Bakhtine-Volochinov 1977), ou de l'ethos comme présentation discursive de soi (Maingueneau 1999, Amossy 2010):

Pas de discours non plus sans ce qu'on pourrait appeler «argumentativité ", ou orientation plus ou moins marquée et plus ou moins raisonnée de l'énoncé qui invite l'autre à partager des façons de penser, de voir, de sentir. En bref, tout discours suppose l'acte de faire fonctionner le langage dans un cadre figuratif ( $\mathrm{Je} »-« \mathrm{tu} »)$, est pris dans la trame des discours qui le précèdent et l'entourent, produit bon gré mal gré une image du locuteur et influe sur les représentations ou les opinions d'un allocutaire. Dans ce sens, l'étude de l'argumentation, et de la façon dont elle s'allie aux autres composants dans l'épaisseur des textes, fait partie intégrante de l'analyse du discours (Amossy $2010: 9$ ).

Ce point de vue pourrait sembler au départ contre-intuitif dans la mesure où il ne correspond pas à ce qu'on entend dans la langue courante par " argumenter ", à savoir "développer une suite d'arguments» (TLFi) ou "justifier, appuyer une thèse, en exposé, etc. par un nombre suffisant d'arguments " (Larousse). Mais il en va de même des autres traits constitutifs du discours : il y a énonciation dans des énoncés débrayés dont la première personne est absente ; dialogisme ne correspond pas à dialoguer dans le sens courant d'échanger des propos avec un interlocuteur; la subjectivité comme "présence du sujet parlant dans son discours » (TLFi) ne signifie pas que se manifeste une expression du moi immédiatement appréhensible; et la présentation de soi apparaît en-dehors des formes où l'on la repère à l'œil nu, dans des discours où le locuteur ne se met pas directement en scène. C'est l'analyste qui en traque les marques, en dégage la construction, en explore les modalités et les fonctions. Ainsi, par exemple, c'est le linguiste qui repère l'effacement énonciatif et les traces gommées de la subjectivité, ou encore dans le cas du dialogisme, la façon dont le discours renvoie à des discours préexistants lorsque l'hétérogénéité n'est pas montrée par des traces claires comme les guillemets ou le discours rapporté (Authier-Revuz 1982). C'est de même l'analyste qui repère les traces de l'argumentation dans les discours qui n'avancent pas d'arguments formels; il en montre "l'argumentativité ", " autrement plus large que ce qui est rangé sous la notion d'argumentation » note ici même Rabatel, qui fait de ce terme un pivot de sa réflexion.

Reprenons l'exemple de Plantin emprunté ici-même par Rabatel: l'information sur l'heure. L'énoncé : «Il est minuit », prononcé par l'un des invités dans un dîner arrivé à sa fin, ne comporte pas de traces d'énonciation, de subjectivité, de dialogisme ou d'argumentativité. En accord avec la pragmatique et au-delà des divergences terminologiques, les théories étendues de l'argumentation comme celles de Grize, d'Amossy ou de Rabatel y voient cependant une dimension argumentative en l'absence de tout argument explicite. L'énoncé invite en effet à penser que l'heure étant tardive, il est temps de rentrer chez soi. Il y a énonciation sans déictiques, subjectivité sans axiologiques ou affectifs, dialogisme sans hétérogénéité montrée. Un sujet parlant s'approprie le langage pour manifester son point de vue en s'appuyant sur une doxa non formulée - minuit est une heure tardive, une heure tardive appelle à aller se coucher ; il transmet sa façon de voir et tente d'orienter des façons de voir et de faire - prendre congé. Cette orientation de l'énoncé vers une conclusion non formulée se met en place grâce à un fonctionnement de la communication fondée sur l'implicite qui ne diffère de ce que les sciences du langage ont exploré, des implicatures de Grice (1979) à la 
présupposition de Ducrot (1972) ou aux topoï selon Anscombre (1995), aux travaux sur l'implicite de Kerbrat-Orecchioni (1986). Nous n'entrerons pas ici dans une analyse plus poussée - qu'il suffise de souligner que des procédures d'induction (ou d'abduction, comme le montre Rabatel sur le cas de la narration) sont activées pour reconstruire ce qui reste dans le non-dit.

11 Cet exemple certes sommaire entendait illustrer le fait que l'argumentativité du discours se laisse saisir en-dehors de la formulation explicite d'une question, d'une thèse et des arguments qui viennent l'étayer. Pour être implicite, elle n'en est pas pour autant absente - à condition d'examiner le discours en situation et en relation avec l'interdiscours, en repérant les marques linguistiques de son inscription. On peut alors - comme le montrent les études réalisées dans ce dossier - saisir dans une grande variété de genres de discours la façon dont non seulement elle oriente des façons de voir un pan de réel ou une situation, mais aussi soulève des questionnements, suscite la réflexion sans nécessairement trancher, manifeste des paradoxes ou des apories.

Dans la mesure où argumenter « n'est pas « attaché [...] à un type spécial de discours ni à l'emploi de technique discursives spécifiques » (Plantin 2016 : 78), on a pu exprimer la crainte - qui n'est certes pas sans fondement - que l'argumentation se dissolve dans l'AD. A cela répond, du côté de ceux qui refusent de s'en tenir à la conception dite restreinte, le désir de rendre compte de l'argumentativité dans toutes ses modalités en se refusant à la dissocier de l'argumentation. Si tous ne s'accordent pas sur l'omniprésence de l'argumentation comme trait constitutif du discours, ils se rejoignent cependant dans le désir d'en rendre compte dans des textes qui la mobilisent en-dehors des schèmes de raisonnement formel et d'élaborer une approche susceptible de l'analyser de façon rigoureuse dans des corpus concrets - nous y reviendrons.

\section{Argumentation et séquence argumentative}

13 La notion de dimension argumentative semble aller à l'encontre des distinctions souvent établies entre des types de texte comme la description, la narration, l'explication et l'argumentation. En réalité, elle ne les remet pas en question, comme Adam l'avait bien souligné dès la première édition de son ouvrage Les textes: types et prototypes (2011 [1992]). Il y mettait en garde contre la confusion entre la «séquence argumentative» qui est une "unité compositionnelle», et l'argumentation dans laquelle il suggérait de voir l'une des fonctions du langage dans le sens de Jakobson - à côté des fonctions phatiques, métalinguistique, poétique, etc. (1992:103).

La séquence argumentative fait chez Adam l'objet d'une étude approfondie fondée sur le schéma de Toulmin (2003 [1958]), qui présente le cheminement menant des données à la conclusion grâce à des règles de passage. D'autres travaux se penchent sur la construction discursive du raisonnement et la séquentialité des textes qui permet d'aboutir à une thèse déterminée, mais en la coupant du cadrage proposé par Adam (séquence argumentative vs. argumentation). Dès lors la démarche qui étudie l'agencement argumentatif du discours exclut de son champ toute parole qui n'est pas agencée de manière à justifier rationnellement une thèse. Pour Micheli (2012), la «visée justificatoire» qui selon lui fonde l'argumentation (plus que la visée de persuasion) « est liée à la question fondamentale de la séquentialité : l'argumentation s'accompagne, au niveau textuel, d'une manière relativement spécifique d'agencer les 
énoncés [...] qui permet de distinguer minimalement l'argumentation d'autres modes d'organisation du discours ».

Le reproche essentiel adressé à l'argumentation dans le discours est alors qu'elle est trop «accueillante» et de ce fait ne permet pas «d'isoler des phénomènes spécifiquement argumentatifs dans le flux des discours » (ibid.). En bref, la conception étendue de l'argumentation qui ne se focalise pas sur la séquentialité est critiquée dans la mesure où elle n'est pas assez discriminante.

\section{Opposition des points de vue et dialogisme}

Mais qu'en est-il d'une condition que certains considèrent comme nécessaire, à savoir l'opposition explicite des points de vue à partir de laquelle le locuteur tente de faire prévaloir le sien aux yeux de l'auditoire? Ainsi pour Plantin, «la communication est pleinement argumentative lorsque la différence est problématisée en une question argumentative et que se dégagent les trois rôles de proposant, d'opposant et de tiers " (2016: 80). Cette approche est contestée ici même par Rabatel, qui considère qu'elle « survalorise » un « modèle dialogal de l'argumentation » issu de la primauté accordée à l'oral aux dépens de l'écrit, modèle qui ne correspond d'ailleurs pas aux conceptions de l'argumentation développées par de grandes théories contemporaines comme celles de Perelman ou de Toulmin. De plus, "quoi de plus "naturel" que de raconter une histoire, d'inférer du sens, et, au total, d'en abduire des conclusions résistantes? Dans cette configuration, un seul discours suffit, et la question problématique n'a pas besoin non plus d'être explicitée, par le locuteur du moins». Et Rabatel ajoute: "De plus, dialogisme et interdiscours aidant, il est souvent très aisé au lecteur ou au destinataire de faire le lien avec des situations antagonistes, des points de vue différents ».

La question du dialogisme est prise en compte par Marianne Doury, qui suit cependant les vues de Plantin sur l'argumentation comme « un mode de gestion discursive du désaccord», une " confrontation entre un discours et un contre-discours» $(2016: 23)$. Elle note en effet qu'il y a « des textes ou des discours dans lesquels toute référence explicite à une opposition est gommée. Ces textes, pourtant, ne prennent leur sens que par rapport à un contre-discours à la lumière duquel émerge leur dimension argumentative » (ibid. : 23). Doury ajoute néanmoins :

Cette invitation à l'élargissement du corpus doit bien sûr rester dans des limites raisonnables, et ne peut pas conduire à étendre à l'infini ses données, dans une application quasi fanatique du principe selon lequel tout discours, peu ou prou, se détermine par rapport à l'ensemble des autres discours existants, ou seulement possibles.

Bien que Doury ne le mentionne pas explicitement, cette position va à l'encontre du rôle prépondérant accordé au dialogisme dans la notion de "dimension argumentative » ou d'« argumentation indirecte». C'est ce que j'ai formulé dans un texte de 2008 en notant :

La position adverse n'a pas besoin d'être présentée en toutes lettres, dans la mesure où la parole est toujours une réponse au mot de l'autre, une réaction au dit antérieur qu'elle confirme, modifie ou réfute :

Toute énonciation, même sous forme écrite figée, est une réponse à quelque chose et est construite comme telle. [...] Toute inscription prolonge celles qui l'ont précédée, engage une polémique avec elles, s'attend à des réactions actives de compréhension, anticipe sur celles-ci, etc. (Bakhtine-Volochinov 1977 : 105). 
Dans cette perspective dialogique, l'argumentation est donc a prioridans le discours [...] condition sine qua non de l'argumentation, même si elle s'y trouve en bonne place. La position restreinte veille à délimiter l'argumentation et à la maintenir dans les frontières d'une définition qui en fait un phénomène distinctif aisément reconnaissable et analysable dans sa spécificité ; elle donne une importance primordiale à sa structure formelle. La position étendue tire les conséquences pour l'argumentation d'un dialogisme généralisé qui situe toute parole dans la circulation des discours, si bien qu'elle la saisit dans son rapport à ce qui se dit et s'écrit dans un espace social donné ; elle manifeste son caractère socio-historique.

\section{Une argumentation sans arguments?}

Si l'argumentation n'est pas à confondre avec la séquence argumentative et ne nécessite pas une confrontation explicite entre des positions contradictoires, qu'en estil de la question de la justification et de l'étayage qui apparaissent dans de nombreuses définitions comme des conditions sine qua non ? Se pose ici la question des arguments à l'aide desquels on soutient une thèse. Peut-on imaginer une argumentation sans arguments? En d'autres termes, y a-t-il argumentation là où il n'y a pas une démarche fondée sur des arguments répertoriés et formellement repérables (comme l'enthymème, l'analogie, l'argument par la conséquence, etc.) qui viennent justifier la position présentée?

21 À ce stade, je voudrais revenir sur un exemple que j'avais analysé dans un article (2007) sur le roman-feuilleton qui se situe autour des années 1846, où Hortense dit de sa mère Adeline Hulot, mariée à un baron ancien général de Napoléon dont les frasques ruinent la famille : « Tu ne sais pas, Lisbeth, eh bien! j'ai le soupçon affreux qu'elle travaille en secret ». L'adjectif affectif mais aussi axiologique "affreux» dramatise l'énoncé et rejaillit sur le travail en lui donnant un sens fortement péjoratif; l'expression «en secret » achève de le marquer du sceau de la honte. La dévalorisation marquée par ces deux qualifications n'est pas d'ordre éthique, mais bien social. A cette époque, travailler pour une femme de la haute société dénonce une pénurie totale, une dégradation qu'on veut dérober aux regards. Cette réplique ne contient pas d'argument formel explicite. Mais elle permet d'inférer du fait que le soupçon est affreux et le travail secret que ce dernier est pour Adeline honteux, et de justifier cette honte par un renvoi aux croyances et valeurs partagées de l'époque, qui correspondent à la doxa de l'auditoire-cible. De ce cas particulier se dégage une norme, une règle, qui entraînent une réflexion plus générale : le travail constitue une déchéance pour toute femme de la bonne société ; la prodigalité et l'irresponsabilité du père de famille mènent à une telle déchéance, comme le montre l'intrigue du récit dans laquelle il faut replonger l'observation d'Hortense. C'est donc dans le discours rapporté - la réplique d'un personnage - que se met en place une dimension argumentative qui n'est pas de l'ordre d'un raisonnement logique formel, mais qui n'en présente pas moins un point de vue déterminé sur le travail (plus particulièrement des femmes) et une leçon sur la prodigalité des maris volages.

Mais il y a plus encore. Pour être interprétée à bon escient, cette réplique doit être replacée dans l'interaction fictionnelle: Hortense s'adresse à Bette, la cousine pauvre

Argumentation et Analyse du Discours, 20 | 2018 
élevée avec Adeline, qui justement travaille pour subsister. Le narrateur s'adresse au lecteur par-dessus la tête de ses personnages. Or Bette, à la page précédente et en fin de chapitre (lieu stratégique du feuilleton), vient de livrer l'une de ses pensées intimes sur le même sujet: «Adeline va, comme moi, travailler pour vivre, pensa la cousine Bette. Je veux qu'elle me mette au courant de ce qu'elle fera... Ces jolis doigts sauront donc enfin comme les miens ce que c'est que le travail forcé ». Le désir de vengeance de la parente pauvre érigée en Opposante maléfique confirme le caractère déshonorant du travail pour une personne du rang d'Adeline. Mais il souligne aussi l'égalisation que le travail établirait entre les deux femmes, toutes deux issues du même milieu populaire et néanmoins promises à un sort si différent. On peut alors se demander si ce retour de fortune est effectivement aussi « affreux » que Hortense veut bien le dire. Le texte ne milite certes pas pour la promotion des travailleurs et l'égalité pour tous et toutes; mais il problématise la question de l'inégalité de la situation faite aux gens du peuple dans la France de 1846, et montre les attitudes d'envie et le désir de revanche qu'elle suscite chez les défavorisés. Le narrateur ne déclarait-il pas au début du roman que « $\mathrm{La}$ cousine Bette [...] appartenait à cette catégorie de caractères plus communs chez le peuple qu'on ne pense et qui peut en expliquer la conduite pendant les révolutions »?

La dimension argumentative du texte se construit ici dans la matérialité du discours, en-dehors de tout schème argumentatif immédiatement formalisable. Elle s'inscrit dans le choix des qualifications et dans le renvoi à une doxa partagée qui permettent au lecteur d'adhérer à un point de vue qui conforte ses valeurs, et de tirer la morale implicite du feuilleton, elle aussi en prise sur son système de croyances. En même temps, par un jeu d'ironie dramatique (le lecteur a accès à un savoir concernant la cousine pauvre qui échappe à la locutrice Hortense, laquelle s'aveugle sur la portée de ses dires), le texte problématise le point de vue consensuel du feuilleton et propose des recoupements qui mettent en cause la question de l'inégalité sociale et du ressentiment populaire qu'elle suscite. Il ne soumet pas une thèse à ses lecteurs, il offre un contrepoint à l'argumentation que développe le récit feuilletonesque en se fondant sur le point de vue dominant et la morale sociale. Semblable construction textuelle soulève implicitement des questions plus qu'elle n'apporte des réponses.

On voit ainsi comment une argumentation peut se construire dans le discours rapporté du texte narratif, et dans la mise en confrontation des paroles des unes et des autres, sans recours à des arguments formels, à des séquences argumentatives ou à des confrontations explicites de positions dont chacune se doit d'être étayée et justifiée.

\section{Dimension argumentative et problématisation}

On voit par ailleurs que l'argumentation travaille ici à problématiser, à proposer un questionnement, plutôt qu'à défendre une thèse. Cette approche est exemplifiée avec brio ici même dans les articles d'Alain Rabatel, qui analyse une fable de La Fontaine, Le loup et l'agneau, et de Tal Sela qui examine l'argumentation dans le roman d'un écrivain d'origine africaine, Mongo Béti. Ils montrent tous deux comment une dimension argumentative, ou une argumentation indirecte dans la terminologie de Rabatel, se construit dans les dessous du texte et propose en filigrane, ici un point de vue différent de la thèse affirmée dans la moralité de la fable («la loi d'airain des rapports de force »), là une problématisation des rapports de l'Afrique noire à la tradition et à la modernité qui contredit le discours doxique du narrateur - les habitants du village 
comme (bons) sauvages. Ainsi, l'analyse de la fable montre "qu'il conviendrait de prendre au sérieux la question du mal, des rapports de force et de réfléchir à la meilleure des façons de traiter cette question, sans se satisfaire de l'idée que les méchants s(er)ont toujours les plus forts ». Celle du roman montre comment la voix des personnages qui traverse celle du narrateur donne l'image complexe d'une société africaine paysanne qui a soif de modernité alors même qu'elle reste enracinée dans la tradition. Pour Rabatel, «c'est par le discours de la narration et l'hétérogénéité discursive que le récit argumente indirectement, parallèlement à l'argumentation directe »; pour Sela c'est également l'hétérogénéité énonciative et discursive telle qu'elle se déploie dans le format de la narration romanesque qui construit la dimension argumentative du texte.

De La Fontaine à Balzac et à Mongo Beti, on peut voir comment le discours problématise une vision doxique plutôt qu'il ne démontre une thèse univoque. Car c'est bien l'une des propriétés de la dimension argumentative qui se construit dans les textes littéraires ici examinés que de procurer un "éclairage " (concept dû à Grize 1990) différent sur le réel et de susciter des questionnements. Dans cette perspective, l'argumentation ne vise pas à faire adhérer à une thèse à travers un raisonnement logiquement valide; par des moyens discursifs variés, elle soumet à l'auditoire un questionnement auquel aucune réponse explicite, et encore moins univoque, n'est fournie. Elle remet en cause des idées reçues, et suscite une réflexion.

Sans doute la notion de "problématisation », prégnante dans mes travaux comme dans ceux de Rabatel, est-elle ici centrale. En l'occurrence, la problématisation consiste ici dans l'acte qui met en question un savoir de sens commun et dévoile des problèmes que recouvrent les évidences. Elle autorise ainsi des points de vue différents, sans pour autant trancher ni se placer dans le paradigme vrai/faux. Il ne s'agit donc pas de la démonstration d'une thèse mais d'un retour sur une doxa partagée et d'un processus de réflexion auquel invitent les mises en perspective du texte.

L'article d'Equoy-Hutin et Lethier sur le dossier d'usager dévoile un autre aspect de la problématisation, liée cette fois à une démarche de coopération et de coordination. C'est celle qui caractérise un genre particulier d'écrit professionnel, le dossier d'usager circulant dans un Centre de Soin, d'Accompagnement et de Prévention en Addictologie (CSAPA). En effet, il participe selon les auteures des genres qui "problématisent, soulèvent des questions et engagent une réflexion sur le mode de la co-construction d'un point de vue collectif». Dans leur travail d'accompagnement d'un usager, les participants aux soins ne tentent pas de persuader l'autre de la véracité d'une thèse : ils inscrivent bien plutôt leur propos dans une interrogation qui s'effectue au sein d'un "processus collectif de questionnements et de prise de décision». "Ainsi», poursuivent les auteures, « la dimension argumentative des textes serait constituée de l'ensemble des contributions explicites ou implicites qui alimentent un cadre de questionnement, en font progresser la densité problématique, voire participent à son orientation vers une conclusion-action particulière ».

L'argumentation apparaît ainsi sous l'une des modalités qu'il importe de prendre en considération, à savoir le processus de co-construction qui se met en place dans une réflexion partagée. L'écrit professionnel est d'autant plus intéressant qu'il ne travaille pas à une co-construction raisonnée, dans le dialogue, de réponses communes à un problème (cas de figure le plus souvent envisagé, notamment dans l'interaction à l'oral). Il offre des interventions écrites dans un style codé de type télégraphique qui 
concrétisent un processus d'accompagnement; elles soulèvent des questions qui orientent des façons de voir et de faire concernant un patient particulier.

\section{Une argumentation sans formulation de problème et sans conclusion?} formulée mais offre des pistes de réflexion sur une question en suspens, ou encore met au jour une question voilée qu'elle invite à (re)penser. Il n'en découle pas pour autant, loin de là, que la dimension argumentative contribue toujours à un processus de problématisation. Bien souvent, au contraire, elle exploite les effets d'évidence qui découlent de l'absence de confrontation explicite pour orienter l'allocutaire vers ses propres façons de voir.

Rabatel souligne que l'argumentation est d'autant plus probante et résistante à la contestation, qu'elle n'affronte pas explicitement la thèse adverse et présente son point de vue sous un jour naturel qui laisse à l'auditoire le soin de dégager les conclusions. Ainsi dans son analyse de l'effacement énonciatif et argumentatif dans Le mort qu'il faut de Semprun, il parlait des descriptions où les «évidences perceptuelles ou conceptuelles » reçoivent une valeur argumentative : le texte ne "démontre » pas, il "montre une situation» (2004:125). Evoquant des "types d'arguments par les faits", Rabatel note: «ils n'ont pas l'air d'arguments, et, s'ils servent à l'argumentation, la charge en échoit au lecteur, qui n'est pas, de ce fait, institué en contradicteur explicite » (ibid.). Alors que l'argumentation directe qui affirme une thèse ou intervient dans un conflit d'opinions génère des contre-discours, l'argumentation indirecte constitue au contraire une démarche d'évitement de la confrontation. La position suggérée est alors d'autant plus probante qu'elle se présente en l'absence de toute position contradictoire, et qu'elle n'est pas énoncée en toutes lettres mais donnée à reconstruire à l'allocutaire qui y adhère d'autant plus facilement qu'il l'a lui-même (re)construite.

Il en résulte que la dimension argumentative peut s'inscrire dans le discours en l'absence d'un problème clairement formulé et d'une réponse en forme de conclusion. Dans La Cousine Bette, le problème de l'inégalité des situations et de l'injustice qui en résulte n'est pas posé. Dans le « livre enrichi » de Delaume - récit en ligne qui exploite les possibilités du numérique - qui traite des asiles d'aliénés, et où la position de l'auteure reste implicite, Amadori montre la façon dont est utilisée la tablette avec son mode énonciatif fictif et ludique pour susciter chez ses lecteurs « des réactions et des émotions susceptibles d'orienter leur opinion » sur l'espace hospitalier sans que celui-ci soit directement discuté. Monte, en analysant le poème de Lorent Gaspar "Les Amandiers ", montre comment il construit sa dimension argumentative en l'absence de toute problématique affirmée. En effet, il soustrait à l'interrogation ce dont il entend imprégner le lecteur. Menant celui-ci «non seulement à croire mais même à éprouver ", il l'invite "à vivre une expérience et pas simplement à construire une représentation du monde». Ici, le raisonnement formel qui vient répondre à une question à laquelle sont apportée des réponses contradictoires cède la place à un « contenu exhortatif » qui « repose sur la communion dans l'émotion ». 


\section{Dimension argumentative ou dimension rhétorique?} également par une réflexion sur la distinction entre argumentation et rhétorique. Ainsi, la différence entre la justification dûment étayée d'une position dans une confrontation de points de vue, et le discours qui oriente des façons de penser, de voir et de sentir, en-dehors de toute confrontation explicite, recoupe la distinction qu'établit Michel Meyer entre argumentation et rhétorique. Pour lui, la première "procède en plaçant la question sur la table, la prenant à bras le corps par des arguments qui s'opposent en faveur de telle ou de telle solution »; la seconde " évacue le problématique en le présentant résolu »; « elle part des réponses comme, si de ce fait même, la question était résolue » (Meyer $2008: 87$ et 85).

'effort de répartir les diverses modalités de l'argumentativité dans des catégories distinctes n'est pas nouveau. Il a été en partie consacré par les découpages disciplinaires : dans les cursus académiques et les revues savantes, on constate souvent que rhétorique et argumentation sont nettement séparées. Cette approche va à l'encontre de la perspective perelmanienne inscrite dans l'intitulé de l'ouvrage pionnier de 1958 : Traité de l'argumentation. La nouvelle rhétorique, lui-même inspiré de la rhétorique argumentative d'Aristote ${ }^{3}$. domaine distinctif est traitée dans ce numéro par Thierry Herman. C'est encore une fois un désir de différenciation et de catégorisation opératoire qui anime l'auteur : "si l'argumentation désigne tous les moyens permettant de provoquer l'adhésion à une thèse, par quel vocable désigner l'enchaînement de la cellule argumentative entre argument(s) et conclusion »?

erman souhaite donc maintenir une définition restreinte de l'argumentation fondée sur l'étayage d'un point de vue. Mais il ne veut pas perdre les bénéfices de la dimension argumentative qui permet d'explorer des discours qui ne relèvent pas du raisonnement formel, comme les tweets de Donald Trump qu'il prend comme corpus. Pour ce faire, il établit une distinction entre l'argumentation, qui comporte des arguments pour étayer une thèse dans un objectif de justification, la question de l'adhésion y étant subsidiaire et la rhétorique où est énoncée une opinion explicite dans laquelle le locuteur s'engage, et à laquelle il tente de faire adhérer son auditoire - l'étayage par des arguments n'y étant pas indispensable. Un exemple tiré de son article : dans « We did it! Thank you to all of my great supporters, we just officially won the election (despite all of the distorted and inaccurate media)», Herman note qu'on trouve une opinion sur les médias, dont l'influence négative n'a pas réussi à faire échouer le candidat - mais elle est "véhiculée sans étayage " tout en appelant à une adhésion de son auditoire. $\mathrm{Ce}$ serait là une «dimension rhétorique » du discours (où le jugement de valeur revêt les apparences du jugement de fait).

Quant à l'éclairage selon Grize, ou la dimension argumentative qui découpe le réel, questionne une situation, à plus forte raison transmet un point de vue à travers une expérience affective sans exprimer d'opinion - elles constituent chez Herman une catégorie à part et ne participent selon lui ni de l'argumentation (il n'y a pas d'étayage) ni de la rhétorique (il n'y a pas d'opinion).

Argumentation et Analyse du Discours, $20 \mid 2018$ 
Dans cette perspective, il ne faudrait pas selon Herman parler d'ethos ou de pathos endehors d'une opinion exprimée. Dans le cas où celle-ci intervient, on reste cependant dans le domaine de la rhétorique et non de l'argumentation. Et en effet, la présentation de soi de l'orateur et le recours au sentiment ne sont pas des raisonnements en forme qui mettent en œuvre des arguments pour justifier une thèse (c'est l'apanage du logos). Ils sont néanmoins opératoires pour provoquer ou renforcer l'adhésion de l'auditoire, comme y insiste la rhétorique d'Aristote qui leur accorde une importance majeure.

Certains articles de ce dossier tentent au contraire de montrer comment l'ethos et le pathos contribuent à susciter des façons de penser et de voir en-dehors de toute opinion exprimée, et rangent ces procédures sous la dénomination de dimension argumentative. Selon Amadori, il y a dans le cas des œuvres numériques désignées sous le nom de «livre enrichi » une tentative de la part de l'auteur comme des éditeurs d'orienter leur réception afin de faire percevoir ces formes d'écriture nouvelles comme pleinement littéraires. L'étude propose une analyse originale de l'ethos éditorial de deux auteures, Frain et Deleaume, qui vient confirmer une image d'auteure préalable extratextuelle: celle d'écrivaines accréditées et charismatiques. Cet ethos éditorial, notion empruntée à Maingueneau, est construit par des éléments techniques - «la couleur, la forme, la taille des caractères, la pagination, la couverture, le sommaire, ainsi que toutes sortes d'allusions éditoriales entretenant un rapport 'dialogique' aux supports de lecture précédents ", qui tous contribuent à orienter l'acte de réception et à conforter le statut littéraire des textes numériques par ailleurs crédités de leur potentiel de nouveauté.

Par ailleurs dans Beauvoir, l'enquête de Frain, «qui se présente comme une enquête, enrichie d'extraits de films, musiques, notes de travail, photos inédites, images d'archives, ayant pour but d'accompagner le lecteur dans les coulisses de l'écriture de Beauvoir in love (Lafon)», l'auteure construit selon Amadori un ethos de "détective nouvelle génération » en fournissant au lecteur des photos, des vidéos, des hyperliens. Elle assume ainsi un rôle de sous-énonciateur en laissant parler ses preuves, ce qui conforte la crédibilité qu'on doit lui accorder dans sa présentation de l'histoire d'amour de Simone de Beauvoir avec le poète américain Nelson Algren comme le plus grand moment de sa vie. "Un tel mode énonciatif, dont la tablette détermine la spécificité [...]», note Amadori, «se met ainsi au service d'une "dimension argumentative" qui tend à se rapprocher de la "visée argumentative ", en mettant en évidence que les deux notions doivent être pensées comme un continuum plutôt que comme deux unités discrètes »

\section{Dimension argumentative et interprétation}

On ne peut éluder ici la question de l'interprétation dans sa relation avec la dimension argumentative du discours. Dans la mesure où celle-ci relève de l'indirection et génère des significations plus ou moins enfouies, elle nécessite un travail de déchiffrement. On peut alors se demander dans quelle mesure la dimension argumentative que l'analyste repère est le résultat d'un déchiffrement qui peut varier selon les récepteurs. En d'autres termes, les significations et points de vue dégagés sont-ils le fait d'une interprétation par définition variable - auquel cas ils ne seraient pas inscrits à même le discours mais constitueraient une construction de lecture par définition aléatoire? 

elle ne peut manquer de se poser pour tout discours qui mobilise l'implicite. Et en effet, elle occupe une partie importante de l'ouvrage consacré à ce sujet par KerbratOrecchioni. Elle se pose avec une acuité toute particulière quand on passe des présupposés qui «sont en principe décodés à l'aide de la seule compétence linguistique", aux sous-entendus qui "font en outre intervenir la compétence encyclopédique des sujets parlants » (Kerbrat-Orrechioni $1986: 41$ ) : l'actualisation des informations portées par l'énoncé porteur d'un sous-entendu est tributaire du contexte et nécessite un «calcul interprétatif ». De façon générale, celui-ci consiste en une opération complexe. Il constitue un travail qui demande de combiner des informations extraites de l'énoncé avec d'autres encyclopédiques (dont on dispose au préalable) « de telle sorte que le résultat se conforme aux lois du discours [...] et aux principes de la logique naturelle»(ibid.: 299). C'est aussi un calcul interprétatif qu'appelle la dimension argumentative des textes - surtout, mais pas uniquement, quand l'indirection et donc la part d'implicite, y est particulièrement flagrante, ou encore dans le cas de textes savamment élaborés dont il faut examiner la structuration, ou la multimodalité, ou qu'il faut déchiffrer dans l'espace numérique en tenant compte de ses particularités comme les hyperliens. Le récepteur se fonde sur des marques langagières (des observables, même si certains peuvent être privilégiés au détriment d'autres), les combine avec un savoir contextuel, un interdiscours (dont la connaissance varie selon les allocutaires), et les intègre dans une construction de lecture respectueuse de la cohérence du texte (mais qui peut varier selon les démarches de reconstruction).

Ainsi, on ne peut nier que dans tous les cas d'indirection fondés sur l'implicite au sens large et sur la complexification du discours, intervient un calcul interprétatif et donc une construction de lecture par définition variable qui assigne au discours certaines significations. Est-ce à dire que la dimension argumentative qui oriente des façons de penser et de voir est le résultat d'une interprétation qui offre une possibilité parmi d'autres, et qu'à la limite, l'analyse de l'argumentativité se confond avec une démarche de construction du sens?

question est certes pertinente ; mais elle n'est pas réservée au cas de la dimension argumentative. N'est-ce pas aussi celui du discours à visée argumentative ? Le travail de Doury (2017) sur le déchiffrement de textes argumentatifs au sens classique effectué par des étudiants le montre bien. Elle écrit ainsi :

Il ne fait aucun doute que les textes et discours argumentatifs sont, sous différents aspects, des données textuelles complexes, dont le sens plein n'est pas accessible via la seule compréhension des mots ni même des phrases qui les composent. C'est un agencement plus global du texte argumentatif qui en génère la pleine signification, agencement qui, pour être saisi, exige de l'interprète qu'il s'appuie sur le sens des mots employés, leur organisation syntagmatique au sein d'énoncés, sur l'organisation énonciative du texte, sur une connaissance de la situation d'énonciation, de l'interdiscours, et plus largement, sur une connaissance du monde.

45 On retrouve ici les éléments du calcul interprétatif énumérés par Kerbrat. Doury ajoute :

L'interprétation d'un discours argumentatif demande également, et peut-être en premier lieu, que l'on s'interroge sur l'intention du locuteur - intention qui, dans une perspective argumentative, est relative à la conclusion qu'il cherche à étayer : interpréter un texte argumentatif, c'est en identifier la conclusion, et montrer 
comment les différents énoncés du texte contribuent, directement ou indirectement, à la rendre plus résistante à la contestation. que toutes deux sont tributaires d'une construction de lecture, dont la difficulté croît au gré de l'importance de l'implicite et de la complexification du discours. Encore fautil préciser qu'il s'agit d'une démarche interprétative qui privilégie tout ce qui, dans le discours, est de l'ordre d'une orientation plus ou moins marquée vers une conclusion, un point de vue, un questionnement - bref, un échange où une tentative d'agir sur l'autre est exercée.

\section{Les observables}

Les articles de ce dossier le montrent bien : la dimension argumentative peut être analysée à partir d'observables qui sont d'ordre divers. Perelman et Olbrechts- 
Tyteca parlaient des «techniques discursives permettant de provoquer ou d'accroître l'adhésion des esprits" (1970[1958]: 5). Ces techniques discursives, ils les répertoriaient aussi bien dans les types d'arguments que dans le choix du lexique, la qualification ou la figuralité. Les avancées des sciences du langage viennent enrichir les données recueillies par la nouvelle rhétorique et permettent de faire une étude fine des textes en procurant des instruments d'analyse plus précis. Ainsi, l'attention au lexique et au choix des termes relève de procédures de nomination ou d'axiologisation qui ont été étudiées en profondeur. Le maniement des connecteurs manifeste des orientations argumentatives, et permet d'intégrer les acquis de la théorie de Ducrot dans l'étude du discours. Les notions de présupposés, de sous-entendus, d'allusions et les diverses formes de l'implicite ont fait l'objet d'analyses fouillées dont les résultats s'avèrent indispensable pour reconstruire une dimension argumentative située dans le non-dit. Les figures du discours, comme la métaphore ou l'ironie, produisent des significations analysées dans les travaux qui en examinent le potentiel discursif et argumentatif (Bonhomme, Paillet et Wahl 2017).

51 On l'a vu, par ailleurs, les travaux sur le dialogisme et l'interdiscours permettent d'éclairer la construction de la dimension argumentative en montrant comment elle se fonde sur un renvoi plus ou moins tacite à une parole antérieure. La réflexion contemporaine sur la doxa enrichit l'étude de l'interdiscours : elle attire l'attention sur les clichés, les formules, les stéréotypes que charrie le discours, tantôt pour transmettre et renforcer leur savoir de sens commun, tantôt pour les retravailler et proposer une vision nouvelle. Les phénomènes d'hétérogénéité énonciative et discursive qui sont au centre de travaux importants mettent en lumière des tensions signifiantes.

52 Il ne s'agit pas ici de dresser un catalogue, mais de souligner que toutes ces procédures discursives, qui interviennent aussi, on l'a dit, dans la mise en mots des arguments, occupent le devant de la scène dès lors qu'en l'absence de raisonnements formels tout se joue dans la trame du texte.

53 C'est ce que montrent les articles de ce dossier, qui examinent la façon dont la dimension argumentative se construit dans des genres très divers. Ceux-ci ne manquent pas d'exercer des contraintes sur les modalités argumentatives. C'est pourquoi ce numéro se propose d'explorer celles-ci dans des cadres génériques divers, dans lesquels la présence de l'argumentativité est plus ou moins reconnue (elle est attendue dans la fable et le tweet mais pas dans le poème lyrique ou l'écrit professionnel d'accompagnement). Il laisse une large place aux genres de discours dans lesquels l'argumentation n'était guère étudiée, afin de sonder les voies de la dimension argumentative. En même temps, les auteurs tentent d'en examiner la rentabilité dans des corpus particuliers. Ils montrent comment une dimension argumentative prégnante se dessine sous la moralité de la fable de La Fontaine (Rabatel) et derrière le discours explicite du narrateur de Mongo Béti (Sela), ou comment elle éclaire le fonctionnement des tweets de Donald Trump quand l'opinion se passe d'étayage (Herman), ou encore ce qu'elle permet de saisir dans un poème lyrique comme ceux de Michaux ou de Jaccottet (Monte), dans un livre enrichi sur Beauvoir ou sur les asiles (Amadori), dans un carnet scientifique en ligne (Mayeur).

54 Last but not least, on a voulu laisser une place aux écrits numériques (Amadori, Mayeur) dont les procédures sont multimodales et qui s'appuient sur les multiples possibilités du Net, pour voir selon quelles modalités innovantes elles permettaient de construire 
une dimension argumentative à travers les nouvelles technologies. Cet aspect est bien mis en valeur par l'article de Mayeur, qui traite des blogs scientifiques en sciences humaines et insiste sur le fait qu'en raison de leur caractère numérique, ils ne recourent pas aux mêmes procédures que celles qui caractérisent les textes écrits et les discours oraux. Mayeur évoque par exemple la «délinéarisation du discours par les hypertextes", ou la "plurisémioticité de l'énoncé intégrant images, vidéos ou animations ». Dans ce contexte, la volonté d'agir sur le lecteur prend une forme très concrète qui appelle à une activation (comme cliquer sur un hyperlien). S'appuyant sur les travaux de Saemmer (2015), l'auteure montre comment le discours scientifique numérique des blogs anticipe des usages en fonction de l'image de l'auditoire (qui est toujours, comme l'a bien noté Perelman, une construction de l'orateur).

De même Amadori, en étudiant le livre enrichi de Delaume qui traite des asiles d'aliénés sans proposer de thèse explicite sur le sujet, mais en construisant une dimension argumentative qui oriente l'approche du lecteur, montre comment la fiction numérique exploite les moyens offerts par les nouvelles technologies. Elles donnent par exemple accès au lecteur non seulement aux journaux des personnages, mais aussi « à des enregistrements de voix, de bruits, à des morceaux de vidéo-surveillance». Amadori parle d'« une performance expérimentale » inspirée du cinéma d'animation et de la «dramatique sonore ». L'examen des éléments qui contribuent à construire une dimension argumentative dans le numérique semble ouvrir une voie féconde et invite à une exploration plus poussée.

Les analyses concrètes de ce numéro sont encadrées par une réflexion théorique approfondie dans les deux textes qui ouvrent le dossier - celui de Rabatel (qui fait suite à d'importants travaux antérieurs) et celui de Herman. La présente introduction espère contribuer à cette réflexion par cet état des lieux succinct qui tente de justifier la notion de dimension argumentative sans éluder les questions parfois difficiles qu'elle soulève, et sans prétendre y apporter des réponses tranchées. Le dossier qui suit permettra à chacun d'évaluer sur pièces les bénéfices ou les défauts d'une conception étendue de l'argumentation qui se veut inclusive.

\section{BIBLIOGRAPHIE}

Adam, Jean-Michel. 2011 [1992]. Les textes : types et prototypes (Paris : Colin)

Amossy, Ruth. 2012 [2000]. L'argumentation dans le discours (Paris : Colin)

Amossy, Ruth. 2005. "The argumentative dimension of discourse ». Van Eemeren, Frans H. \& Peter Houtlosser (eds). Practices of Argumentation (Amsterdam: John Benjamins), 87-98

Amossy, Ruth. 2007. « Les récits médiatiques de grande diffusion au prisme de l'argumentation dans le discours : le cas du roman feuilleton », Belphégor, Idéologie et stratégies argumentatives dans les récits imprimés de grande consommation. XIXème - XXIème siècles 54 (en ligne) 
Amossy, Ruth. 2008. « Argumentation et Analyse du discours : perspectives théoriques et découpages disciplinaires ", Argumentation et Analyse du Discours, mis en ligne le 06 septembre 2008

Ruth Amossy, 2009. "Argumentation in Discourse : A Socio-discursive Approach to arguments », Informal Logic 29:3, 252-267

Amossy, Ruth. 2010. La présentation de soi. Ethos et identité verbale (Paris : PUF)

Amossy, Ruth \& Roselyne Koren. 2009. « Rhétorique et argumentation : approches croisées », Argumentation et Analyse du Discours [En ligne].

Angenot, Marc. 2008. Dialogues de sourds : traité de rhétorique antilogique (Paris : Mille et une nuits) Anscombre, Jean-Claude \& Oswald Ducrot. 1988. L'Argumentation dans la langue (Liège : Mardaga) Anscombre, Jean-Claude, éd. 1995. Théorie des topoï (Paris : Kimé)

Authier-Revuz, Jacqueline. 1982. « Hétérogénéité montrée et hétérogénéité constitutive : éléments pour une approche de l'autre dans le discours ", DRLAV 26, 91-151

Bakhtine, Mikhail (v. n. Volochinov). 1977. Le Marxisme et la philosophie du langage (Paris : Minuit) Benveniste, Emile, 1966. Problèmes de linguistique générale, vol. 1 (Paris : Gallimard)

Benveniste, Emile, 1974. Problèmes de linguistique générale, vol. 2 (Paris : Gallimard)

Bonhomme, Marc, Anne-Marie Paillet \& Philippe Wahl (éds). 2017. Métaphore et argumentation (Louvain-la-Neuve : Academia)

Doury, Marianne. 2016. Argumentation. Analyser texte et discours (Paris : Colin)

Doury, Marianne. 2017. « Les écueils de l'interprétation de l'argumentation », Les sciences du langage et la question de l'interprétation (aujourd'hui), communication au Colloque de l'ASL, 2 décembre 2017

Ducrot, Oswald, 1972. Dire et ne pas dire. Principes de sémantique linguistique (Paris: Hermann)

Grice, H.P., 1979, « Logique et conversation », Communications 30, 31-56

Grize, Jean-Blaize, 1990. Logique et langage (Paris : Ophrys)

Kerbrat-Orecchioni, Catherine, 1980. L'Énonciation de la subjectivité dans le langage (Paris : Colin)

Kerbrat-Orecchioni, Catherine, 1986. L'Implicite (Paris : Colin)

Maingueneau, Dominique. 1999. « Ethos, scénographie, incorporation », Images de soi dans le discours. La construction de l'ethos, Amossy, R. (éd.) (Lausanne : Delachaux et Niestlé)

Meyer, Michel. 2008. Principia Rhetorica (Paris : Fayard)

Micheli, Raphaël. 2012. « Les visées de l'argumentation et leurs corrélats langagiers : une approche discursive ", Argumentation et Analyse du Discours [En ligne], 9 | 2012, mis en ligne le 15 octobre 2012, consulté le 09 avril 2018. URL : http://journals.openedition.org/aad/1406 ; DOI : $10.4000 /$ aad. 1406

Micheli, Raphael. 2010. L'émotion argumentée. L'abolition de la peine de mort dans le débat parlementaire (Paris : Le Cerf)

Perelman, Chaim \& Lucie Olbrechts Tyteca. 1970. [1958] Traité de l'argumentation. La nouvelle rhétorique (Bruxelles : Éditions de l'Université de Bruxelles) 
Plantin, Christian. 2011. Les bonnes raisons des émotions. Principes et méthode pour l'étude du discours émotionné (Berne : P. Lang)

Plantin, Christian. 2016. Dictionnaire de l'argumentation une introduction aux études d'argumentation (Lyon : ENS)

Rabatel, Alain. 2004 Argumenter en racontant (Bruxelles : De Boek)

Rabatel, Alain. 2014 « Quelques remarques sur la théorie argumentative de la polyphonie » Arena romanistica, 14, 204-222

Rabatel, Alain. 2016. «En amont d'une théorie argumentative de la polyphonie, une conception radicale de l'énonciation comme énonciation problématisante », Verbum XXXVIII, 1-2, 131-150

Toulmin, Stephen E. 2003 [1958]. Uses of argument (Cambridge University Press)

\section{NOTES}

1. Ces références sont données à titre indicatif uniquement. Les textes de Rabatel théorisent et exemplifient à de nombreuses reprises l'argumentation indirecte, qui est synonyme de dimension argumentative.

2. Je remercie Christian Plantin de la discussion très éclairante que j'ai eue avec lui à propos de cette distinction, et Roselyne Koren de ses remarques et suggestions.

3. Cf. l'introduction que j'ai rédigée avec Roselyne Koren dans Argumentation et analyse du discours 2 (2009).

\section{AUTEUR}

\section{RUTH AMOSSY}

Université de Tel aviv, ADARR 